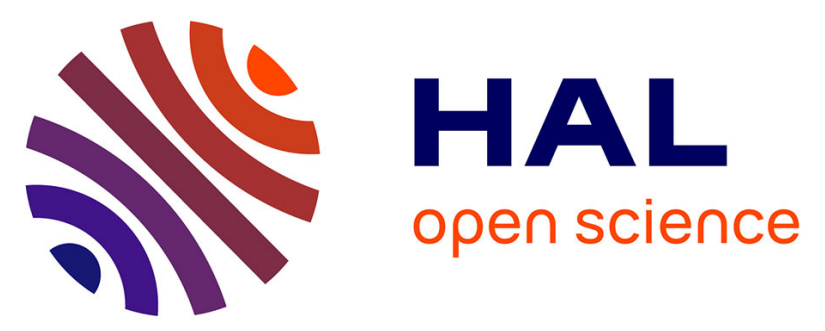

\title{
Novel concept of a low-power high-volume microfluidic actuator: theory of operation and experimental characterization
}

Quentin Lavigne, Nicolas Terrier, Guillaume Noetinger, Duc-Duy Tran, Simon Kulifaj, Pascal Kleimann, Patrick Pittet, Bruno Berge

\section{To cite this version:}

Quentin Lavigne, Nicolas Terrier, Guillaume Noetinger, Duc-Duy Tran, Simon Kulifaj, et al.. Novel concept of a low-power high-volume microfluidic actuator: theory of operation and experimental characterization. Sensors and Actuators A: Physical , 2019, 10.1016/j.sna.2019.03.018 . hal-02072918

\section{HAL Id: hal-02072918 \\ https://univ-lyon1.hal.science/hal-02072918}

Submitted on 22 Oct 2021

HAL is a multi-disciplinary open access archive for the deposit and dissemination of scientific research documents, whether they are published or not. The documents may come from teaching and research institutions in France or abroad, or from public or private research centers.
L'archive ouverte pluridisciplinaire HAL, est destinée au dépôt et à la diffusion de documents scientifiques de niveau recherche, publiés ou non, émanant des établissements d'enseignement et de recherche français ou étrangers, des laboratoires publics ou privés.

\section{(c)(1) $\$$}

Distributed under a Creative Commons Attribution - NonCommerciall 4.0 International 


\title{
Novel concept of a low-power high-volume microfluidic actuator: theory of operation and experimental characterization.
}

\author{
Quentin Lavigne ${ }^{a}$, Nicolas Terrier ${ }^{b}$, Guillaume Noetinger ${ }^{a}$, Duc-Duy Tran ${ }^{a}$, Simon \\ Kulifaj $^{\mathrm{a}}$, Pascal Kleimann ${ }^{\mathrm{b}}$, Patrick Pittet $^{\mathrm{b}}$ and Bruno Berge ${ }^{\mathrm{a}^{*}}$ \\ aLaclarée, ENS-Lyon, 9 rue du Vercors, 69007 Lyon, France. \\ bInstitut des Nanotechnologies de Lyon (INL), Université de Lyon, CNRS UMR 5270, Villeurbanne, \\ France. \\ * corresponding author
}

A novel concept of microfluidic actuator based on a slack metallized film confined between two parallel grid-electrodes separated by a gap is presented. The film is loosely attached such that it can move freely between the two grid-electrodes. Upon voltage application between the metallized film and one grid-electrode, electrostatic attraction tends to press the metallized film on the grid, thus pushing the fluid through the grid. This evolution happens through a film fold travelling across the cell width. Samples using a replication of grid-electrodes manufactured by silicon anisotropic etching have been assembled. An equilibrium theory of operation and an experimental characterization with measurements of exchanged liquid volumes, differential pressures, capacitance and applied voltage are presented, both agreeing within +/$15 \%$. It will be shown that actuators with an active zone of $8 \mathrm{mmx} 45 \mathrm{~mm}$ and a gap of $0.25 \mathrm{~mm}$ can move $100 \mu$ l against differential pressures of about $200 \mathrm{~Pa}$ with a $100 \mathrm{~V}$ power supply at $100 \mathrm{~Hz}$ consuming few tens of $\mathrm{mW}$. The only moving part is the deformable film and the actuator is silent when filled with liquids. The new concept also supports an accurate capacitance monitoring of the exchanged volumes. The actuation can be reversible by connecting one or the other grid electrode. This new pumping mechanism has been primarily designed for exchanging a given volume of two insulating fluids of different refractive indices, in order to activate ophthalmic variable eyeglasses for presbyopia correction. Nevertheless, the same concept can be extended to continuous flow pumping.

Keywords: microfluidic actuator - electrostatic micro-pump - active eyeglasses

\section{Introduction}

Microfluidics is a very active field of research and addresses a wide range of potential applications, opening perspectives of new integrated devices to deliver drugs[1-3], to analyze and manipulate chemical components[4], to make embedded optical systems[5] or to build soft robots[6,7]. Nevertheless, a wider diffusion of such microfluidics devices will rely on the improvement of the fabrication techniques and of their cost price as well as on their easiness of use. 
Micro-pumps are components of key importance for fluids actuation in such systems. A wide spectrum of operating principles have been proposed for micro-pump implementation[8-10]. Electrostatic actuation allows a fast response time with a low power consumption. Zengerle et al proposed an implementation where a diaphragm is displaced by electrostatic force between two electrodes[11]. The main limitation of this approach is that the actuation force steeply decreases when the distance between the diaphragm and the electrodes increases. Machauf et al tackled this problem by suggesting the use of the dielectric properties of the working fluid to reinforce the pumping[12]. The evolution of electrostatic micro-pumps has also been accompanied by the development of different types of membranes and geometries. Different peristaltic pumps operate with membranes composed of silicon plates diced in a wafer and coated with Parylene or other insulating materials [13-16]. The recent development of ionic polymer materials which can be electro-actuated entails the fabrication of new kind of pumps[17,18]. Ghazali et al have designed a pump implementing a soft dielectric elastomer[19].

In these different approaches, the deformable membrane is clamped and stretched. Moreover, when pumps are designed for large volume displacement, they are operated at high frequency, raising a dissipation issue in the case of viscous fluids[8]. In a series of papers following the original work of Shikida et al. [20], several authors have proposed micromechanical systems based on the deformation of an S-shaped metallic ribbon actuated by electrostatic forces. These works served to make valves, RF or optical switches[21-23]. We propose in this paper a novel concept of lowpower high-volume microfluidic actuator based on a flexible S-shaped metallized polymer film, which adresses the isssues raised by previoulsy proposed pump architectrures for large fluid volume displacements applications[24]. 

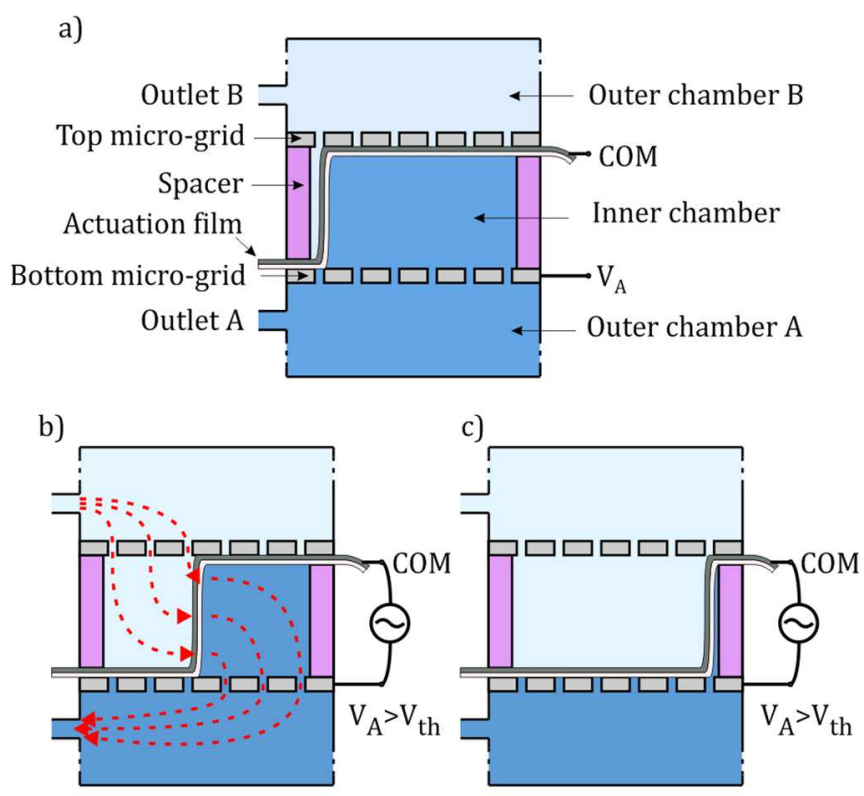

Figure 1 a) Architecture and b), c) principle of operation of the proposed electrostatic pump. The pump implements a metallized actuation film placed slack with a "S-shape" at the interface between two insulating fluids. Two micro-grids defined the limits of the inner chamber and are used as actuation electrodes. When the actuation voltage applied between the film and one electrode reaches a threshold, the film fold moves because of electrostatic attraction on the biased electrode. When the biasing is lower than this threshold, the film moves back to its initial position due to counter-pressure.

In this work, our main goal was to develop a microfluidic actuator changing the focal power of a two-fluid filled adaptive ophthalmic lens. This ophthalmic lens will be used in the context of presbyopia correction[25]: The actuator enables to push/pull the two fluids having two different index of refractions. These fluids are connected to two thin chambers within an ophthalmic lens separated by a thin deformable membrane. The microfluidic actuator is then used to change the optical power by regulating the amount of the high index fluid versus the low index fluid in the ophthalmic lens[25][24]. The microfluidic actuator (pump) for such an application needs to exchange a significant volume $(\sim 100 \mu \mathrm{l})$ of two fluids (oils) within $1 \mathrm{~s}$ with a power consumption as low as possible. In this paper, we describe the design and principle of operation of this novel valve-less actuator concept in a first section. Then, we present the material and methods used to fabricate and to characterize actuator prototypes. In the third section, the experimental results are reported and discussed in the fourth section.

\section{Design and principle of operation}

\subsection{Principle of operation}

Figure 1 illustrates a unidirectional pumping cycle on a 2D cross section of the proposed pump architecture. A thin bendable polymer film (with a typical thickness of few microns), named in the following the "actuation film", metallized on a single side, 


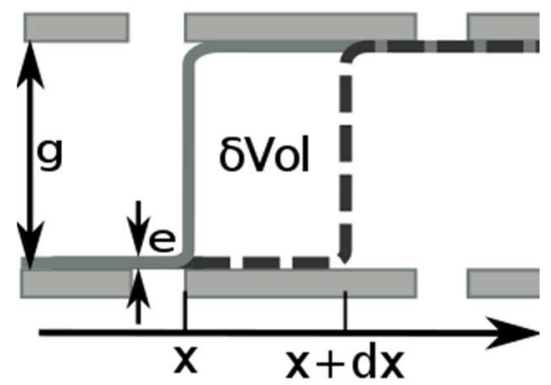

Figure 2 Pumped volume $\delta V o l$ for an infinitesimal translation of the of the film fold from position $x$ to $x+d x$

is maintained between two parallel conductive micro-grids separated by a gap, $g$ (typically within the 100-350 $\mu \mathrm{m}$ range). These micro-grids allow fluid communication between the inner-chamber and outer-chambers. The fluid management is based on electrostatic forces, and requires that the fluid which is between the actuation film and the grid electrode (dark blue on Figure 1 ) is insulating (no ionic charges).

The film is set slack and loose, allowing its free motion between the two micro-grids. Two spacers are used to place the extremity of the film in contact with the bottom and top micro-grids, respectively. Thus, the cross section of the film can be divided in three regions: one in contact with the bottom grid, another in contact with the top grid, and a S-shape transition zone as illustrated in Figure 1. This S-shape transition zone is one of the key feature of this actuation concept and will be called "the film fold". The actuation of the pump is realized by applying a voltage between the actuation film (COM Electrode) and bottom micro-grid (Electrode $A$ ) resulting in the displacement of the film towards this micro-grid due to electrostatic attraction. More precisely the displacement of the actuation film is done through a rolling travel of the film fold laterally (from Figure 1 a to $1 \mathrm{c}$ ). The parts of the film which are in contact with the top or bottom grids have no velocity relatively to the grids during this travel.

If there is a positive differential pressure between A and B outlets, the actuation film is pressed against the top electrode, as shown in Figure 1a. When a sufficiently high actuation voltage is applied between actuation film and bottom micro-grid, the electrostatic force prevails over the pressure forces and the film is attracted against bottom micro-grid, as shown in Figure 1c. This transition happens at a threshold voltage, called $V_{t h}$, which depends on the differential pressure (see details in section B Theory).

Below (resp. above) $V_{t h}$ the situation is depicted by Figure 1a (resp. 1c) the film fold being blocked at the left (resp. right) spacer. Figure $1 \mathrm{~b}$ depicts the transient state at $V_{t h}$, in the middle of the film fold travel (see video in supplementary materials). When the actuation voltage is no more applied on the electrodes, the film fold slides back to its initial position (Figure 1a). When there is no differential pressure imposed from outside, activation of the voltage between COM and grid A electrode will induce a positive differential pressure to push liquid $A$ at the expense of liquid $B$. 
This mode of operation is the simplest mode, qualified as unidirectional, the actuator being able only to actively push the fluid $A$. Another mode enables this pump to work in a reversible mode. When the pump is operated in such a bi-directional mode, an external voltage is applied between the actuation film (COM electrode) and either the bottom micro-grid (Electrode $A$ ) or the top micro-grid (Electrode B). Connecting the voltage source to Electrode $A$ will deliver positive differential pressure between outlets $A$ and $B$. By connecting the voltage source to electrode $B$ the reverse effect will be induced. It has to be noted that for this mode of operation, it is necessary to insulate the top micro-grid to prevent any shortage with the metallized side of the actuation film.

During a actuation cycle, the rolling of the film fold modifies the relative volumes of the inner-chambers, thus expelling or sucking out fluids. It has to be noted that the proposed pump is working at constant overall volume, i.e. when a volume of fluid $A$ is expelled out from chamber $A$, the same volume of fluid $B$ is input in chamber $B$.

It is worth noticing that the micro-grids as well as the outer-chambers are designed to optimize the fluidic resistance of the pump. This is required in the case of the actuation of adaptive ophthalmic lens. A more compact implementation of this novel pump can be considered by replacing the micro-grids by two plates and placing the outlets at the level of the inner-chambers as holes through the spacers for instance.

\subsection{Theory of operation}

The key parameters governing the actuator operation are the actuation voltage $V$, the differential pressure, $\Delta P=P_{B}-P_{A}$, the displaced volume of fluid, $\mathrm{Vol}$ as well as the capacitance between the two electrodes $C_{p}$ (i.e. the bottom micro-grid and the actuation film).

When the actuation voltage $V$ exceeds a threshold voltage $V_{t h}$, the film fold moves as previously explained. In order to find thermodynamic equilibrium, let's consider an infinitesimal translation of the film fold from $x$ to $x+d x$ as shown in Figure 2 . The electrostatic energy variation is given by:

$$
d E_{L}=\frac{1}{2} \cdot V^{2} \cdot d C_{p}
$$

The capacitance $d C_{p}$ can be estimated by the variation of the surface of film which is in close contact with the electrode:

$$
d C_{p}(x)=c_{S} \cdot L_{y} \cdot d x
$$

where $c_{S}$ is the surface capacitance of the dielectric film and $L_{y}$ the length of the micro-grid electrode (orthogonal to Figure 2). 
Using the planar infinite capacitor approximation the surface capacitance of the film, $c_{s}$ can be expressed as:

$$
c_{S}=\frac{\varepsilon_{r} \cdot \varepsilon_{0}}{e}
$$

where $e, \varepsilon_{r}, \varepsilon_{0}$ are the film thickness, the relative dielectric constant and the vacuum permittivity, respectively.

Thus, the electrostatic energy change can be expressed as:

$$
d E_{L}=\frac{1}{2} \cdot V^{2} \cdot c_{S} \cdot L_{y} \cdot d x
$$

The work $\delta W$ for expelling the volume of fluid $\delta V o l=g . L_{y} . d x$ against the differential pressure $\Delta P$, is given by:

$$
\delta W=g \cdot L_{y} \cdot d x \cdot \Delta P
$$

The thermodynamics equilibrium condition is achieved for the threshold voltage $V_{t h}$ when $d E_{L}=\delta W$.

In this condition, based on equations (4) and (5), we get:

$$
\Delta P=\frac{1}{2} \cdot \frac{c_{S}}{g} \cdot V_{t h}^{2}=\frac{1}{2} \cdot \frac{\varepsilon_{r} \cdot \varepsilon_{0}}{e \cdot g} \cdot V_{t h}^{2}
$$

It has to be noted that equation (6) differs from the expression of the electrostatic pressure for conventional electrostatic actuators which is [26]:

$$
\Delta P \approx \frac{1}{2} \cdot \frac{\varepsilon_{r} \cdot \varepsilon_{0}}{g^{2}} \cdot V_{t h}^{2}
$$

This difference shows the benefit associated with the slack film, since $(e g) \ll g^{2}$ and thus a much lower voltage can be used for actuation as compared with the voltage requirement to develop the same electrostatic pressure with the conventional approach (stretched deformable membrane). Equation 6 also emphasizes the important role of the gap $g$, meaning that one of the important feature of this microfluidic actuator is the confinement of the film between the two grids.

The total volume of the fluid that can be expelled is directly related to the inter-grid volume and can be expressed as:

$$
V o l_{\max }=g \cdot L_{x} \cdot L_{y}
$$

where $L_{x}$ is the film width.

The capacitance variation $\delta C_{p}(x)$ is governed by the surface film in contact with the active electrode which is pressed against the active electrode and is given by:

$$
\delta C_{p}(x)=\frac{c_{S}}{g} \delta \operatorname{Vol}(x)
$$


The set of equations (6), (8) and (9) can be used for the design of the microfluidic actuator.

It is worth mentioning that we assumed here a constant S-shape of the film fold during actuation. Within this assumption, the exact shape of the film fold has no effect on the actuation threshold voltage: indeed this detailed shape gives rise to a mechanical energy stored in the fold. As this energy is the same for both positions $x$ and $x+d x$, its cancels out in equation (6). The same argument applies also to the electrostatic energy stored in the stray-field (edge effects) at the vicinity of the fold, such that (6) appears as an exact solution of the thermodynamics problem, with our assumptions.

The threshold actuation voltage $V_{t h}$ in equation (6) does not depend of the current film position $x$. That means that for a constant differential pressure, $\Delta P$, once the threshold voltage is reached, the translation of the film fold starts and travels all the way towards the final film position (shown in Figure 1c).

In this constant pressure mode, the volume of fluid which is exchanged cannot be controlled by the voltage. However, it should be noted that, according to equation (9), the variation of the capacitance $C p$ is proportional to the displaced volume and thus an electronic measurement of the capacitance can be considered to precisely monitor the exchanged volume.

Equation (6) can be seen either as the threshold voltage for actuator transition as a function of the externally imposed differential pressure, or as the actuator (pump) output pressure as a function of the externally applied voltage.

Equation (4) contains a slight approximation, and more rigorously $c_{s}$ should be replaced by the difference in film-grid surface capacitances for the two extreme positions of the film, "down on the floor" and "up to the ceiling". As the gap is about 100 times larger than the actuation film thickness, this last capacitance will be negligible compared to the other, thus validating equation (4).

\subsection{Considerations on the design of the grid-electrodes}

As already mentioned, the use of micro-grids as electrodes is motivated by the need to optimize the fluidic impedance for actuating adaptive ophthalmic lenses. Hence some attention should be paid on the micro-grid design. The pressure losses through the micro-grids should remain negligible as compared to pressure losses in the rest of the fluidic circuit and obviously to the differential pressure capability of the actuator. As explained in ref [26], pressure losses through perforated plates depends on the size and density of holes in the micro-grid (i.e. the porosity of the screen) as well as the plate thickness relatively to the hole diameter.

The pressure losses through the micro-grid, $\Delta P_{\text {grid }}$, can be estimated as: 


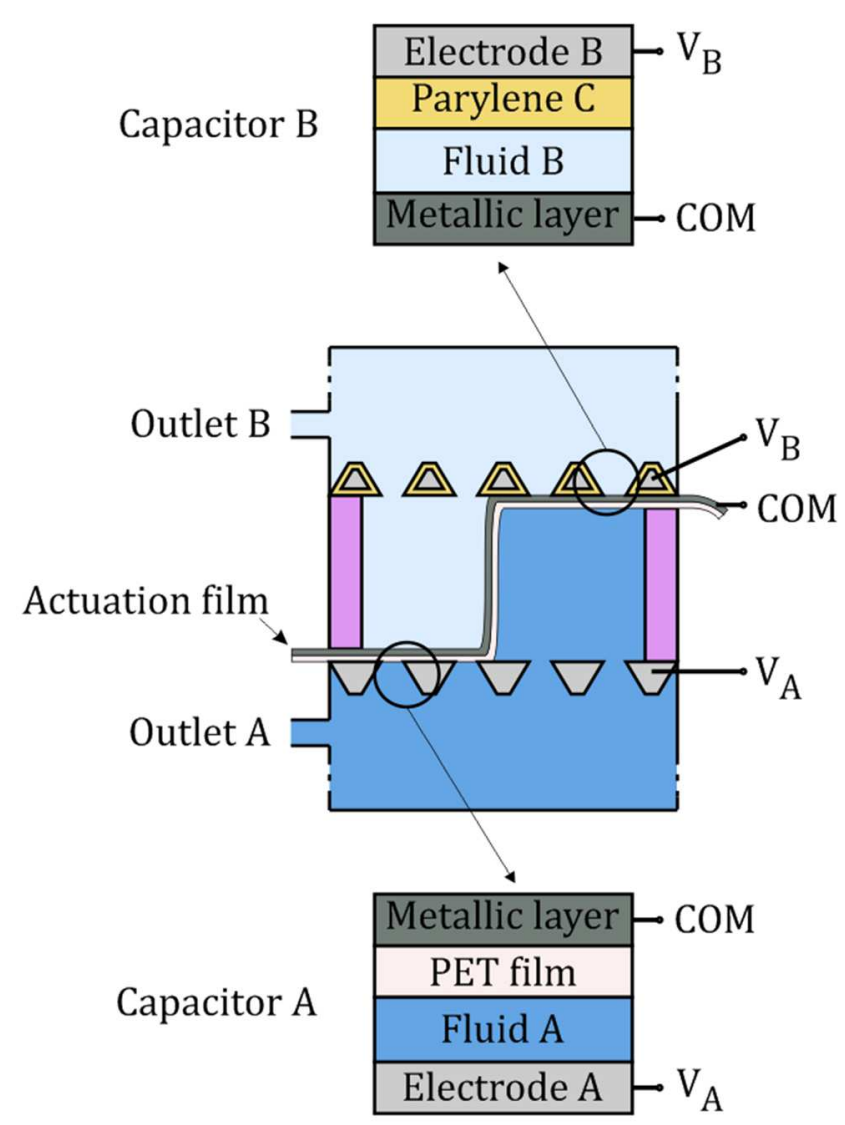

Figure $3 \mathrm{Bi}$-directional implementation of the micro-pump: a) pump cross section; stacking of the different layers constituting b) capacitor $A$ and c) capacitor $B$.

$$
\Delta P_{\text {grid }}=k\left(\frac{\rho v^{2}}{2}\right)
$$

where $k$ is the pressure loss coefficient, $v$ the bulk-mean velocity and $\rho$ the fluid density.

According to equation (6), the electrostatic actuation force is directly proportional to the surface capacitance $c_{S}$. A special attention should be paid during the micro-grid design and fabrication to avoid any short scale roughness, burr or dust particles on the grid surface. Such defects would prevent an intimate contact between the film and the grid, and thus would reduce the surface capacitance.

It is noted that the porosity (size and density of holes) of the micro-grids induces a reduction of the active surface of the electrode resulting in a decrease of the corresponding capacitance. Thus, there is a trade-off between low fluidic impedance requiring high porosity and electrostatic efficiency requiring low grid porosity.

We adopted a grid microfabrication process based on anisotropic wet etching of silicon, which is particularly advantageous. Such a process allows the realization of pyramidal holes with smooth surfaces. Thus, the grid apertures are much smaller on the side of the micro-grid in contact with the film (ensuring good electrostatic efficiency) while they are larger on the other side (minimizing the pressure losses 


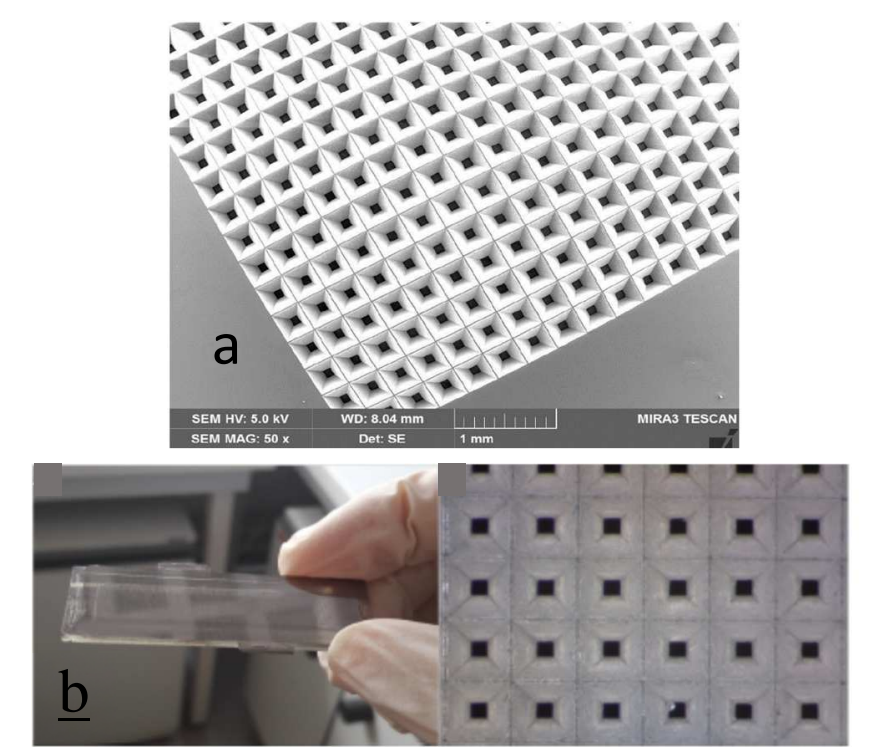

Figure 4 a) SEM image of silicon micro-grid; b) image of the polymer replica

through the micro-grid due to viscous forces). This microfabrication process is detailed in the following section.

\subsection{Considerations on the electrical actuation of the S-shaped film}

From an electrical point of view, the micro-pump behaves as a variable capacitance and the resulting fluid output differential pressure is proportional to voltage squared as shown in Equation (6). Either DC or AC actuation voltages could thus be implemented. Nevertheless, with DC actuation voltages, a small conductivity of the fluid due to the presence of residual ionic species could adversely affect the electrostatic force (this concept of actuation requires an insulating liquid). For this reason, we used low frequency AC voltage (100-200 Hz). For a sinusoidal shaped drive signal, the dissipated power is given by:

$$
P_{\text {ower_dissipated }}=2 \pi D f C_{p} V^{2}
$$

where $f$ is the switching frequency and $D$ is the dissipation factor, a dimensionless coefficient which is measured by the LCR meter.

\section{Materials and methods}

\subsection{Micro-fabrication of the silicon micro-grids}

The microfabrication process was realized at Nanolyon clean room facility. We first deposited a $1.4 \mu \mathrm{m}$ photoresist layer ( $\mathrm{AZ}^{\circledR}$ 5214, Microchemicals $\mathrm{GmbH}$, Germany) onto a $200 \mu \mathrm{m}$ thick <100> silicon wafer with $\mathrm{Si}_{3} \mathrm{~N}_{4}$ films on both sides. The photoresist layer was patterned by UV-lithography. A controlled $\mathrm{SF}_{6} / \mathrm{Ar}$ plasma reactive ion etching is used to transfer the pattern in the silicon nitride film. This patterned $\mathrm{Si}_{3} \mathrm{~N}_{4}$ film served as hard mask for $\mathrm{KOH}$ silicon wet etching. Apertures in the 


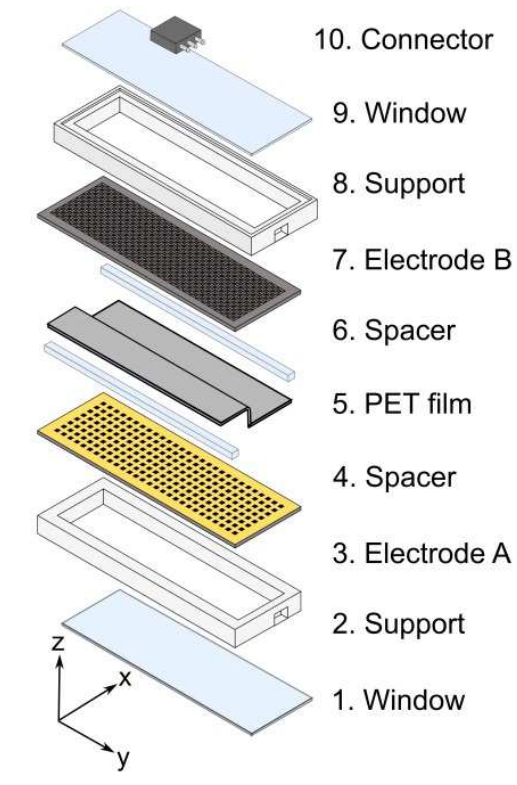

Figure 5 Exploded view of the electrostatic micropump

film produced inversed truncated pyramidal structures in the silicon wafer since the etching rate along the $<111>$ crystallographic planes is much slower than along the $<100>$ ones. A V-groove is also realized along the grid outline during the wet etching and is used for an accurate and easy cleaving of the individual grids.

A SEM image of one micro-fabricated grid is shown in Figure 4a.

We have also fabricated micro-grids by replication of a silicon master in a polymer as shown in Figure $4 \mathrm{~b}$ (The replication method is not detailed here). A conductive coating ( $5 \mathrm{~nm}$ of $\mathrm{Cr}$ and $30 \mathrm{~nm}$ of $\mathrm{Au}$ ) is deposited on each replicated grid by e-beam evaporation (EVA300, Alliance Concept, France).

It is worth mentioning that we do not observe any difference between silicon and polymer micro-grids when they are implemented in the proposed micro-pump (the pump performances depend on the micro-grid geometry but not on the micro-grid material). Thus, characterization studies were realized indifferently on both microgrid types.

As already mentioned, for bidirectional pump implementation, it is necessary to insulate the top micro-grid by a conformal layer in order to prevent any short-circuit between the micro-grid and the metallized side of the film (see Figure 3a). In our case, we have used Parylene $C$ as a conformal insulator which is widely used to insulate electrical connexions in MEMS[27]. Moreover, this material has excellent dielectric and mechanical properties and is compatible with the fluids which will be used in the micropump[28].

\subsection{Microfluidic actuator assembly}


The exploded view for the proposed electrostatic microfluidic pump is shown in Figure 5.

The pump implements two micro-grids also used as electrodes ( 3 and 7 ), which are glued on 3D printed mechanical supports ( 2 and 8 ). These supports define the external body of the pump as well as the geometry of the pump outer-chambers. Spacers ( 4 and 6 ) realized in a PET film by Xurography are used to maintain both lateral ends of the film, against the bottom and top micro-grids, respectively (defining the gap g). These spacers also define the volume of the inner-chamber. The actuation film (5) is made of a $2 \mu \mathrm{m}$ thick commercial PET film with sputtered aluminium on one side.

It is worth mentioning that during the assembly this film is mounted unstretched. The absence of stretching is decisive because it ensures the maximal performance of the pump allowing smooth motion of the film fold during pump actuation.

Two or three pins electrical connector (10) are mounted for uni- and bi-directional operations, respectively. Silver conductive paint is used to connect the electrodes and the film to the output pins.

\subsection{Characterization setup}

We have developed a PC-controlled characterization bench allowing step by step validation and characterization of the pump prototypes in terms of film-grid capacitance $C p$, micro-pump gap $g$, exchanged volume $\mathrm{Vol}$, externally applied differential pressure $\Delta P$ and applied voltage $V$. Prototype testing is carried out either with air or with oil as fluids. This bench as shown in Figure 6 is composed of four main

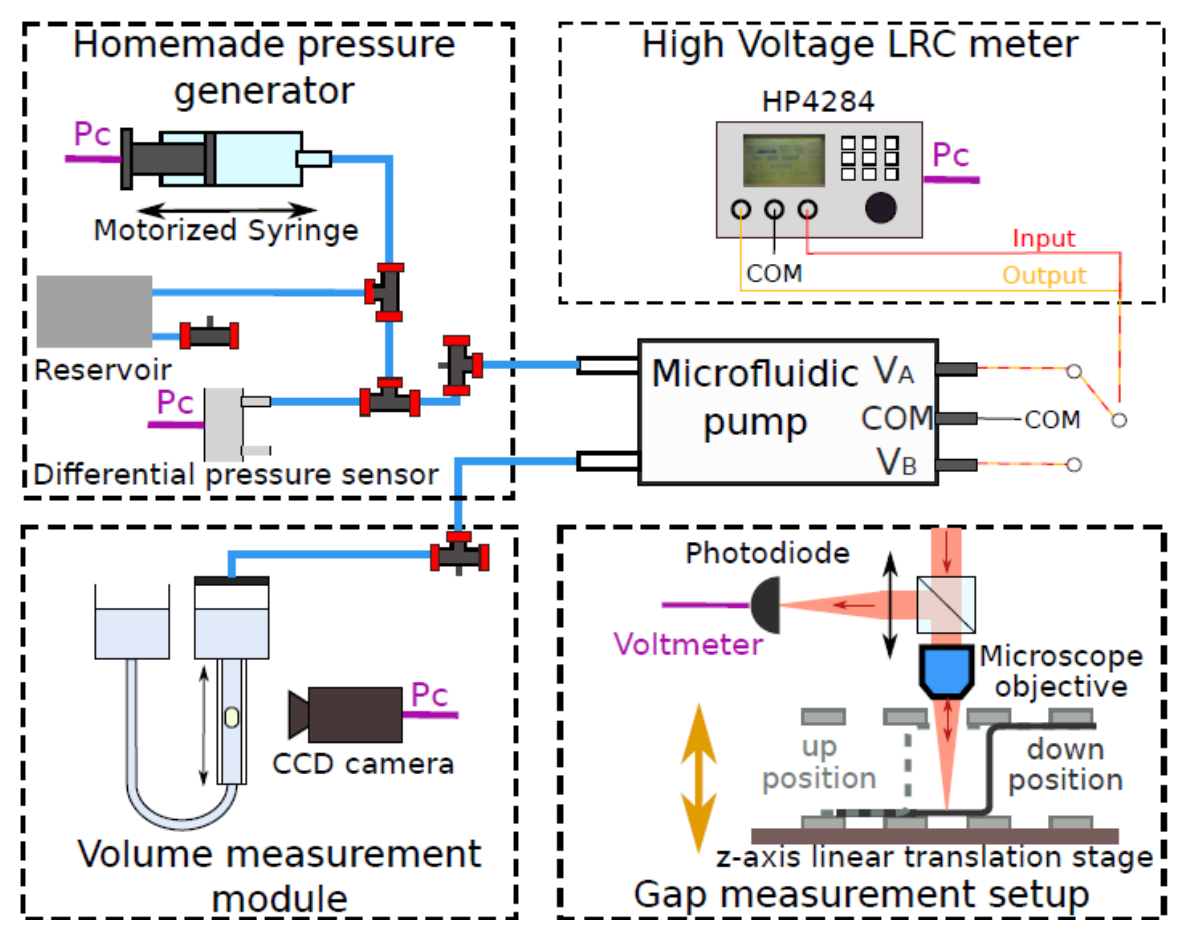

Figure 6 The characterization bench with its four main modules 
modules:

- A homemade pressure generator regulating the differential pressure $\Delta P$. The pressure generator consists of a motorized syringe able to inject/retract well defined volumes of air volume (within $+/-2.5 \mathrm{ml}$ ) in a large volume chamber $(400 \mathrm{ml})$. A differential Omega pressure sensor PX163 monitors the corresponding generated differential pressure.

- An impedance meter (HP4284 LCR meter).

- A homemade volume measurement module. Briefly, the principle is based on the measurement of the displacement of a silicon oil droplet in a calibrated borosilicate pipe segment filled with water.

- A homemade gap measurement setup. The principle consists in measuring the position of the metalized film in the pump chamber through a hole in the top microgrid. This is achieved by using a laser beam focused on the film using a long distance microscope objective (NIKON ELWD 20X/0.40 A) working in a confocal microscope configuration.

\section{Experimental}

About 45 functional pump prototypes have been assembled and used for this characterization study. They implement:

- $2 \mu \mathrm{m}$ thick PET actuation film (Steinerfilm ${ }^{\circledR} \mathrm{T}$, Germany),

- two PET spacers of $175 \mu \mathrm{m}$,

- two micro-grids of $8 \times 45 \mathrm{~mm}^{2}$ active surfaces with a matrix of square holes of $100 \times 100 \mu \mathrm{m}^{2}$ with a pitch of $350 \mu \mathrm{m}$. It is noted that with the implemented prototype assembly process, we observed significant variations of the gap from one prototype to another (it ranged from $220 \mu \mathrm{m}$ to $350 \mu \mathrm{m}$ ).

The total inner chamber volume is $\sim 110 \mu$ f for a typical gap of $g=300 \mu \mathrm{m}$, while the outer-chambers volume is about 10 times larger.

Due to manual and delicate assembly of these prototypes, we observed a significant dispersion of their characteristics which prevent any statistical analysis of the measured data.

\subsection{Film-grid capacitance: $\boldsymbol{C p}$}

The capacitance $C p$ between the metallic side of the film and each micro-grid has been measured as a function of the differential pressure, $\Delta P$ on a bi-directional actuator prototype. A volume of air was injected at a known pressure in chamber $B$ while chamber $A$ was let at atmospheric pressure. After pressure stabilization in chamber $\mathrm{B}$, the capacitance $C p$ was measured with the LCR meter by using a $10 \mathrm{~V}$ and $200 \mathrm{~Hz}$ probing signal.

Each side of the pump was characterized in a separated experiment. The probing voltage is well below the actuation voltage threshold making electrostatic forces negligible. 


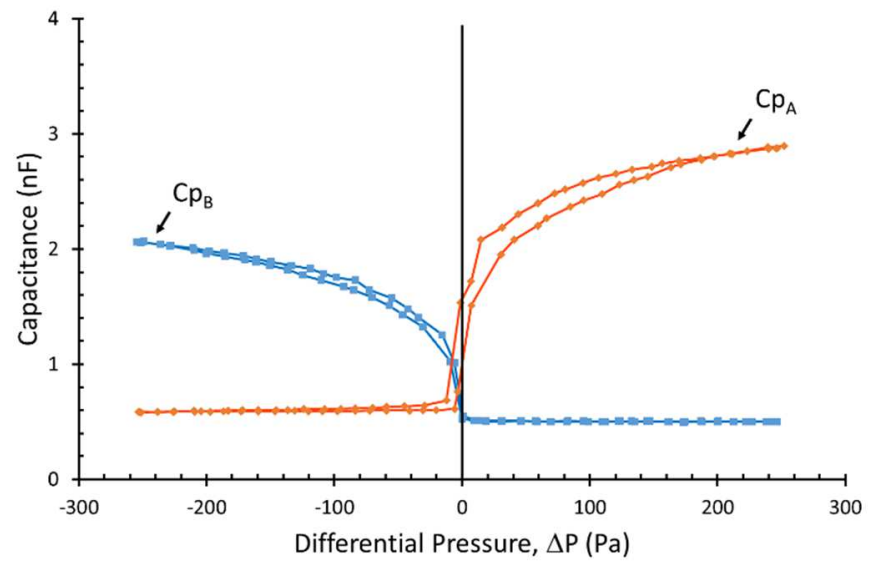

Figure 7 Evolution of $C p A$ and $C p B$ measured over a cycle of differential pressure $\triangle \mathrm{P}$.

Figure 7 shows the evolution of $C p A$ and $C p B$ measured over a cycle of differential pressure $\Delta P$.

When the differential pressure pulls the film away from the micro-grid, the capacitance is minimal with a quasi-constant value corresponding to the small surface of the film which is maintained in contact with the micro-grid under the spacer.

When the differential pressure presses the film towards the micro-grid, the capacitance reaches a plateau when most of the film is in contact with the micro-grid. Upon cycling the differential pressure, $\Delta P$, we observed an hysteresis in the transition zone of $10 \mathrm{~Pa}$ for $C p A$ and $5 \mathrm{~Pa}$ for $C p B$, respectively. The raise of capacitance at low pressures and the difference of variation of capacitance between the two electrodes presumably come from the air residual layers (see also the section on measurement of surface capacitance). It is noted that the dissymmetry was reduced when liquids (oils) are used instead of air (not shown on figure).

\subsection{Surface capacitance: $\boldsymbol{c}_{\boldsymbol{s}}$}

The surface capacitance $c_{s}$ has been measured with the actuation film lying on a standard glass slab $\left(50 \times 16 \mathrm{~mm}^{2}\right)$ coated with a $\mathrm{Cr} / \mathrm{Au}$ conductive layer. This characterization study has been carried out on 7 different glass slabs with 10 independent measures per slab. The LCR meter was set up with $70 \mathrm{~V} / 200 \mathrm{~Hz} A C$ voltage. We measured $c_{S}=8.5 \frac{\mu F}{m^{2}}$ which is of the same order of magnitude of the film surface capacitance determined with equation (3), i.e. $c_{s}=14.2 \frac{\mu \mathrm{F}}{\mathrm{m}^{2}}$. We attribute this difference to a slab of air, which adds up a serial surface capacitance: using oil instead of air usually increases the surface capacitance, a usual phenomenon in capacitor impregnation. This also confirms the need for a smooth film and electrode roughness. Moreover, the measured value of $c_{s}$ was found to be more reliable than its theoretical value and was used for the actuator design.

\subsection{In-situ measurement of the actuator gap: $g$}


The confocal set-up has been used for the in-situ evaluation of the gap $g$ between the two micro-grids. The gap has been measured over $24 \mathrm{X}-\mathrm{Y}$ locations spread over the grid (ranging from $X=0$ to $8 \mathrm{~mm}$ and from $Y=0.9$ to $18 \mathrm{~mm}$ ). It was evaluated to be $g=240 \mu \mathrm{m}$ with a standard deviation of $30 \mu \mathrm{m}$ for this prototype, which shows a good homogeneity of this gap for a given prototype. As mentioned earlier, we observe much larger gap variations from prototype to prototype. For instance, the gap of the prototype studied in Figure 8 was $\sim 340 \mu \mathrm{m}$. These large sample-to-sample variations are attributed to the residual thickness of the glue, motivating the implementation of the in-situ gap measurement module.

Figure 8 shows the displacement of the film between the two micro-grids, when it is actuated by a pressure. The width of the transition between the two plateaux of the curve of Figure 8 can be used to estimate the minimal pressure required for the film displacement: it requires $\sim 10-20$ Pa of pressure for its full displacement. This pressure

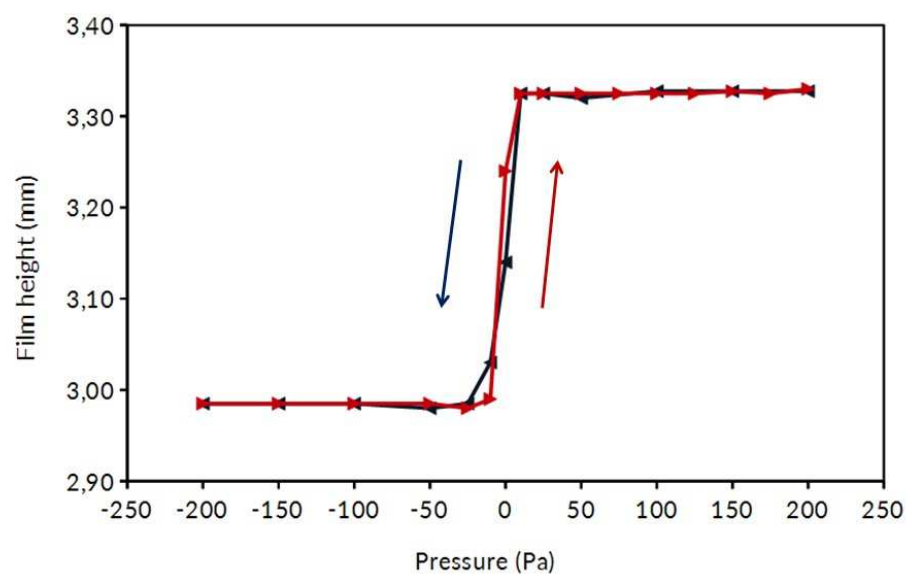

Figure 8 Film height change when the film is actuated by differential pressure.

can be neglected as compared to the generated differential pressure of the fluid $(\triangle \mathrm{P}=200 \mathrm{~Pa})$. Moreover, there is no significant hysteresis when the film is displaced from one position to the other one and vice-versa.

\subsection{Pumping volume: $\mathrm{Vol}$}

This experiment was carried out by using the testing bench configured with the pressure-generator and measurement-volume modules connected to $A$ and $B$ pump chambers, respectively. The position of the droplet as well as the film-grid capacitance, $C p$ were simultaneously acquired for different differential pressures $\Delta P$, applied between the two chambers.

Results are reported in Figure 9 and show that this prototype is able of pumping up to Vol $115 \mu \mathrm{L}$. This is consistent with the volume of fluid which is contained in the inner chambers between the film and the micro-grid (for the measured gap of 240 $\mu \mathrm{m}$, and for the prototype grid surface of $8 \times 45 \mathrm{~mm}^{2}$, we were expecting a volume of Vol $\sim 90 \mu \mathrm{l})$. 
In Figure 9, we observe a linear relationship between the capacitance $C p$ and the pumped volume $\mathrm{Vol}$, which is in line with equation (9). It allows an experimental characterization of the coefficient $\delta C p / \delta V o l$. Moreover, it confirms that the pumping cycle can be followed in real time by monitoring the capacitance $C p$.

\subsection{Electrical actuation of the pump}

The capacitance $C p$ has been used to monitor the status of the pump when it is electrically actuated by an AC voltage. The actuator is operated while keeping a



Figure 9 Simultaneous measurements of capacitance $C p$ and volume of pumped fluid, $V o l$.

constant externally applied differential pressure.

In Figure 10, we observe an abrupt transition of the capacitance $C p$ (corresponding to the film fold displacement) when a threshold voltage, $V_{t h}$ is reached. Ideally the film fold should travel all the way across the cell at Vth. In practice, there are always small imperfections and the transition is slightly smeared, as can be seen in the supporting video. As shown by equation (7), this threshold actuation voltage, $V_{t h}$ depends of the differential pressure $\Delta P$. In addition, we observe in Figure 10 a hysteresis on the threshold voltage which will be discussed in the next section.

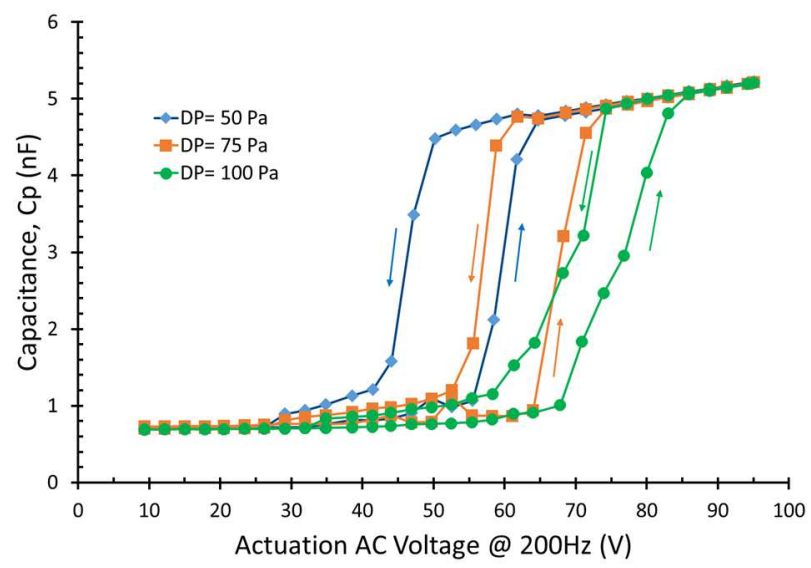

Figure 10 Capacitance variations for upward/downward ramping cycles of the actuation voltage 
It is also possible to estimate in Figure 10, the expelled volume of fluid during the pumping cycle. It is proportional to the variations of the capacitance $\Delta C p$ according to equation (9) and to the curves of Figure 9. It was evaluated to be $\sim 110 \mu \mathrm{l}$.

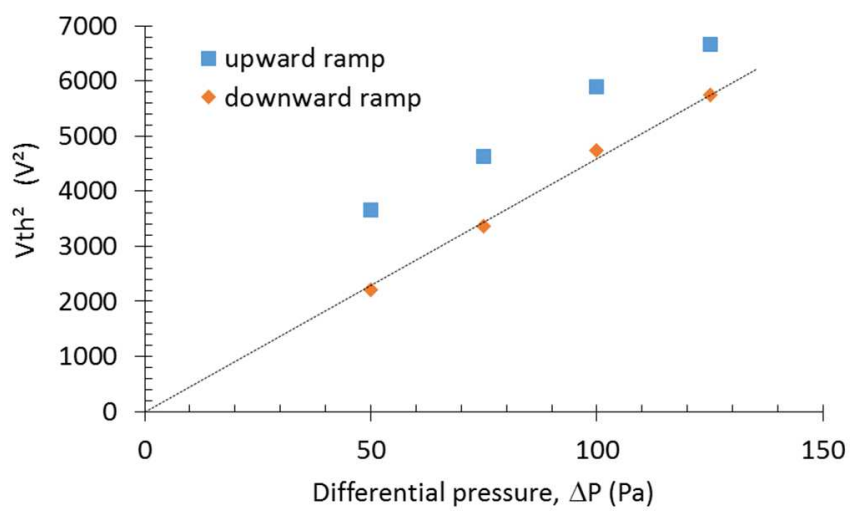

Figure 11 Square actuation threshold voltage versus the differential pressure for downward and upward ramping cycles.

\subsection{Actuator fatigue testing}

To evaluate the effects of fatigue on its performances, the actuator was continuously operated over a period of 3h30 with 1200 actuation cycles when loaded by a $100 \mathrm{~Pa}$ back pressure, with air as fluid. The actuation voltage was toggled between $20 \mathrm{~V}$ and 100V.

During this test, the capacitance of the actuator was monitored in real time. We observed no significant change of the measured capacitances in both states (in terms

Table 1: Preliminary fatigue test results

\begin{tabular}{|c|c|c|}
\hline Studied cycles & $\begin{array}{c}\text { Measured capacitance for an } \\
\text { actuation voltage of 20V (nF) }\end{array}$ & $\begin{array}{c}\text { Measured capacitance for an } \\
\text { actuation voltage of 100V (nF) }\end{array}$ \\
\hline$[0-10]$ & 0.94 & 4.40 \\
\hline$[100-110]$ & 0.95 & 4.41 \\
\hline$[500-510]$ & 0,93 & 4.41 \\
\hline$[1190-1200]$ & 0.92 & 4.42 \\
\hline
\end{tabular}

of mean value and standard deviation) over the full test duration as shown in table 1. This observation confirms that the properties of the S-shaped actuation film does not change during this preliminary fatigue test. 
Table 2: Summary of experimental measurements in comparison with expected data from the thermodynamics theory.

\begin{tabular}{|c|c|c|c|c|c|}
\hline Type & Item & Unit & $\begin{array}{l}\text { Design data } \\
\text { according to } \\
\text { theory }\end{array}$ & $\begin{array}{l}\text { Measured } \\
\text { Data }\end{array}$ & Method \\
\hline \multirow{3}{*}{ Geometry } & Electrode area & $\mathrm{mm}^{2}$ & 360 & & \\
\hline & gap g & $\mu \mathrm{m}$ & $220-350$ & 245 & $\begin{array}{l}\text { In-situ measurement using } \\
\text { confocal set-up }\end{array}$ \\
\hline & Vol_max & $\mu \mathrm{l}$ & $110 \mu \mathrm{g} \mathrm{g}=300 \mu \mathrm{m}$ & 115 & from Figure 9 \\
\hline \multirow{3}{*}{ Capacitance } & Surface capacitance $c_{s}$ & $\mu \mathrm{F} / \mathrm{m}^{2}$ & 14.2 & 8.5 & $\begin{array}{l}\text { Actuation film deposited on a } \\
\text { metallized glass slab }\end{array}$ \\
\hline & \multirow{2}{*}{$\Delta \mathrm{Cp} \_$max } & \multirow{2}{*}{$\mathrm{nF}$} & & 4.3 & from Figure 10 \\
\hline & & & & 4.8 & from Figure 9 \\
\hline \multirow{4}{*}{$\begin{array}{l}\text { Pump functional } \\
\text { response }\end{array}$} & $\overline{C_{s} / g}$ & $\mu \mathrm{F} / \mathrm{m}^{3}$ & & 0.035 & \\
\hline & $\Delta \mathrm{P}^{2} \mathrm{~V}^{2}$ & $\mathrm{~Pa} / \mathrm{V}^{2}$ & 0.017 & 0.022 & from Figure 11 \\
\hline & $\delta C p / \delta V o l$ & $\mathrm{nF} / \mu \mathrm{l}$ & 0.034 & 0.044 & from Figure 9 \\
\hline & $\Delta$ Cp_max/ $\Delta$ Vol_max & $\mathrm{nF} / \mu \mathrm{l}$ & 0.034 & 0.036 & from Figure 9 \\
\hline
\end{tabular}

Data in bold correspond to best estimate

\section{Discussion}

Data of Figure 10 can be processed to determine the actuation threshold voltages versus the differential pressure for upward and downward voltage ramping cycles. We plot in Figure 11, the extracted square actuation threshold voltage against the differential pressure.

For downward curve, the linear relationship predicted by equation (6) is observed. However, there is an unexpected offset for the upward ramping as well as different slopes for upward and downward cases.

Table 2 shows a summary of experimental measurements in comparison with expected data from the thermodynamics theory.

As shown in Table 2, the proposed set of equations gives a good description of the pump operation and can be used for the pump design. The functional response agrees with theoretical prediction within +/- $15 \%$ accuracy. We consider this agreement as satisfactorily, considering manual assembly techniques and experimental imprecisions. It has to be noted that one expects from theory that $d C p / d V o l$ is equal to $c_{s} / g$ and equal to 2 times the $d P / d\left(V^{2}\right)$. This relationship was observed experimentally.

Table 2 confirms that the proposed architecture allows actuation with voltages significantly lower than conventional electrostatic pump ( 10 times lower for the implemented dimensions). The experimental results show also that operating at a gap 
as high as $250 \mu \mathrm{m}$ enables to displace $100 \mu \mathrm{l}$ against $200 \mathrm{~Pa}$ counter-pressure using a few tens of $\mathrm{mW}$, in-line with applications requiring large volume management as the ophthalmic lens.

Regarding actuation power, Equation 11 enables to estimate its order of magnitude. Figure 10 shows that the maximum actuator capacitance was $\sim 5 \mathrm{nF}$, with a measured dissipation factor of 0.028 for the side where the PET is the insulator and 0.079 on the side where the Parylene is the insulator (not shown in Table). At $100 \mathrm{~Hz}$ drive frequency and $100 \mathrm{~V}$ applied, the resulting power dissipated in the actuator, Power_diss, is about $0.47 \mathrm{~mW}$ (resp. $1.26 \mathrm{~mW}$ ) for the PET (resp. Parylene) side. These values correspond to the power dissipated inside the pump only, and do not take into account the power dissipated in the driver IC. If one wants to have an order of magnitude of the efficiency, one can estimate the delivered peak power when the pump is pushing the fluid volume outside against a counter pressure, given by:

Power_fluid $=\Delta \mathrm{P} *$ Volume/tau

, where Power_fluid is the mechanical power transferred to the fluid by the pump, $\Delta \mathrm{P}$ is the counter pressure, Volume is the transferred fluid volume and tau is the time to deliver this volume. In our pump implementation, this leads to $22 \mu \mathrm{W}(110 \mu \mathrm{l}$ pushed against $200 \mathrm{~Pa}$ in $1 \mathrm{~s}$ ). The resulting efficiency, Power_fluid/Power_dissipated, is about $4,6 \%$ (resp. $1.7 \%$ ) for the PET side (resp. Parylene side). If one includes the power dissipated in the driver part, the efficiency falls to about $0.04 \%$, showing that most of the losses come from the driver.

Figure 10 reveals the presence of hysteresis in the actuation film movements under applied voltage, whereas Figure 8 and 9 reveal that when the actuator is operated by pressure only (no or little voltage) the actuation film moves with no or little hysteresis. This suggests that the hysteresis observed in Figure 10 is linked with electrostatic effects: any pure mechanical adhesion of the film on the grids or any rigidity of the film fold can be discarded for the explanation of the observed hysteresis. Nevertheless an adhesion of the film due to the electrostatic pressure cannot be excluded at this stage.

Hysteresis could be also attributed to the presence of holes in the micro-grids, generating a spatial modulation of electrostatic forces. In order to derive an order of magnitude of this effect, we will consider two threshold voltages (see Figure 12): A threshold voltage $\boldsymbol{V}_{\boldsymbol{t h} \mathbf{1}}$, when the film fold does not meet any row of holes (left part of Figure 12) and a threshold voltage $\boldsymbol{V}_{\boldsymbol{t h 2}}$, when the film fold meets a row of holes (right part of Figure 12). $\boldsymbol{V}_{\boldsymbol{t h} \mathbf{2}}$ is expected to be larger than $\boldsymbol{V}_{\boldsymbol{t h 1} \mathbf{1}}$ because the presence of holes reduces the active surface of the electrode and thus, it is necessary to apply a higher threshold voltage to reach a sufficient electrostatic force for film actuation. 
Quantitatively $\boldsymbol{V}_{\boldsymbol{t h} \mathbf{1}}$ could be estimated by Equation (6) with $\boldsymbol{c}_{\boldsymbol{s}}$ corresponding to a plain electrode (no hole), where $\boldsymbol{V}_{\boldsymbol{t h} \mathbf{2}}$ could be estimated with a reduced surface capacitance $\boldsymbol{c}_{\boldsymbol{s}}\left(\mathbf{1}-\frac{\boldsymbol{a}}{\boldsymbol{b}}\right)$ due to the holes, where $\boldsymbol{a}$ and $\boldsymbol{b}$ are the hole size and period respectively. This would lead to the upward (resp. downward) ramping threshold voltage corresponding to the $V_{t h 2}\left(\operatorname{resp} . V_{t h 1}\right)$.

According to equation (6), the threshold actuation voltage $\boldsymbol{V}_{\boldsymbol{t h 2}}$ follows:

$$
\Delta P=\frac{1}{2} \frac{c_{s}\left(1-\frac{a}{b}\right)}{g} V_{t h 2}^{2}
$$

leading to the hysteresis estimate as:

$$
\Delta V_{t h}=V_{t h 2}-V_{t h 1}=\sqrt{\frac{2 g \Delta P}{c_{s}}}\left(\sqrt{\frac{1}{1-\frac{a}{b}}}-1\right)
$$

Assuming $a<<b$, equation (13) can be simplified as:

$$
\Delta V_{t h} \sim \frac{a}{2 b} V_{t h 1}
$$

Equation (14) reflects the underlying hypothesis of an electrostatic root cause for the hysteresis, this last one being proportional to the applied voltage $\boldsymbol{V}_{\boldsymbol{t h} \mathbf{1}}$. This finding is however in contradiction with Figure 10 and 11 where the hysteresis seems to decrease with voltage. A full understanding of the Hysteresis phenomenon will require further studies, including $3 \mathrm{D}$ effect as the mechanical rigidity of the film fold (right part of Figure 12).

However, it should be noted that there are several ways to get around the actuator's hysteresis in practice:

- driving using the monitoring of the capacitance: In this mode of operation, the linearity of the capacitance with exchange volumes suppresses the hysteresis (see Figure 9).

a) Side view
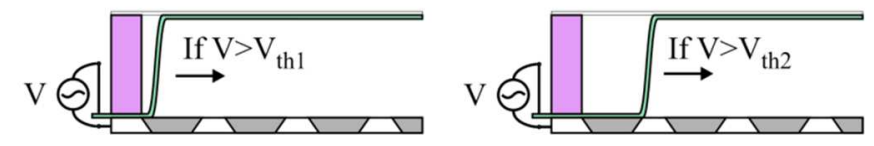

b) Top view of the bottom micro-grid electrode

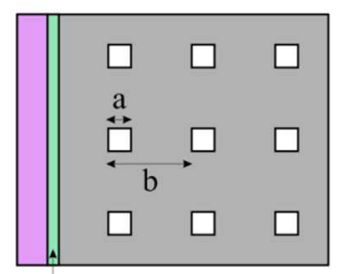

Actuation film

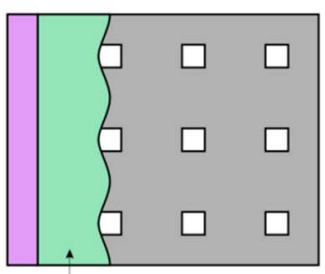

Actuation film

Figure 12 Impact of the holes of the micro-grids during upward and downward ramping of the actuation voltage 
- using discrete electrodes instead of a single conductive grid, and driving each sector by either 0 or the maximum voltage.

It is not possible to mention all possible variants of the actuator, all sharing the two most important features:

1. presence of a slack film instead of stretched membranes

2. spatial confinement of this slack film, the gap g playing a central role in the actuation efficiency (equation (6)).

For example grids could be replaced by solid plates, with fluid passages through the lateral spacers, thus suppressing most dead volume related to the chambers. Also the core concept of the actuator being very thin, cascading several stages, in series or in parallel is easy to implement in order to match with particular application specifications.

\section{Conclusion}

A novel concept of microfluidic pump has been presented based on a slack metallized film trapped between two grid-electrodes separated by a gap. Samples using a replication of silicon grid-electrodes manufactured by anisotropic etching have been assembled. An equilibrium theory of operation and a full experimental characterization with measurements of exchanged liquid volumes, differential pressures, capacitance and applied voltage have been presented, both agreeing within +/- $15 \%$. It has been shown that sample pumps with an active zone of $8 \mathrm{~mm} \times 45 \mathrm{~mm}$ and a gap of $0.25 \mathrm{~mm}$ can move more than $100 \mu \mathrm{l}$ against differential pressures of about $200 \mathrm{~Pa}$ with a $100 \mathrm{~V}$ power supply at $100 \mathrm{~Hz}$, using very low electrical power. It has been shown that the hysteresis of the actuator probably originates from electrostatic effects. Further investigations which will be carried out in order to better understand the origin of hysteresis.

The mechanism is primarily designed for insulating fluids but could be extended to water based solution, by using a unidirectional pump with an insulating fluid (air or oil) at the active side. The basic principle of such actuator is open to many variants and dimensions. The prototypes shown in this paper have been designed to pump a given amount of liquid in the context of activating ophthalmic variable eye-glasses, but the same principle could be extended to continuous flow pumping by using additional valves.

\section{Conflicts of interest}

Bruno BERGE is an executive of the company Laclarée, a company developing adaptive eyeglasses for presbyopia correction. Quentin LAVIGNE was employee of Laclarée, Guillaume NOETINGER and Duc-Duy TRAN have been trainees in Laclarée. 


\section{Acknowledgements}

ENS-Lyon is acknowledged for physics and chemistry labs support and for access to mechanical 3D printer.

\section{References}

[1] F. Amirouche, Y. Zhou, T. Johnson, Current micropump technologies and their biomedical applications, Microsyst. Technol. 15 (2009) 647-666. doi:10.1007/s00542009-0804-7.

[2] A. Nisar, N. Afzulpurkar, B. Mahaisavariya, A. Tuantranont, MEMS-based micropumps in drug delivery and biomedical applications, Sensors Actuators B Chem. 130 (2008) 917-942. doi:10.1016/J.SNB.2007.10.064.

[3] N.-C. Tsai, C.-Y. Sue, Review of MEMS-based drug delivery and dosing systems, Sensors Actuators A Phys. 134 (2007) 555-564. doi:10.1016/J.SNA.2006.06.014.

[4] R.-J. Yang, C.-C. Liu, Y.-N. Wang, H.-H. Hou, L.-M. Fu, A comprehensive review of micro-distillation methods, Chem. Eng. J. 313 (2017) 1509-1520. doi:10.1016/j.cej.2016.11.041.

[5] N.-T. Nguyen, Micro-optofluidic Lenses: A review, Biomicrofluidics. 4 (2010) 31501. doi:10.1063/1.3460392.

[6] D. Han, H. Gu, J. Kim, S. Yokota, A bio-inspired 3D-printed hybrid finger with integrated ECF (electro-conjugate fluid) micropumps, Sensors Actuators A Phys. 257 (2017) 47-57. doi:10.1016/J.SNA.2017.02.002.

[7] M. Wehner, R.L. Truby, D.J. Fitzgerald, B. Mosadegh, G.M. Whitesides, J.A. Lewis, R.J. Wood, An integrated design and fabrication strategy for entirely soft, autonomous robots, Nature. 536 (2016) 451-455. doi:10.1038/nature19100.

[8] D.J. Laser, J.G. Santiago, A review of micropumps, J. Micromechanics Microengineering. 14 (2004). doi:10.1088/0960-1317/14/6/R01.

[9] P. Woias, Micropumps-past, progress and future prospects, Sensors Actuators B Chem. 105 (2005) 28-38. doi:10.1016/J.SNB.2004.02.033.

[10] B.D. Iverson, S. V. Garimella, Recent advances in microscale pumping technologies: a review and evaluation, Microfluid. Nanofluidics. 5 (2008) 145-174. doi:10.1007/s10404-008-0266-8.

[11] R. Zengerle, A. Richter, H. Sandmaier, A micro membrane pump with electrostatic actuation, in: [1992] Proc. IEEE Micro Electro Mech. Syst., IEEE, 1992: pp. 19-24. doi:10.1109/MEMSYS.1992.187684.

[12] A. MacHauf, Y. Nemirovsky, U. Dinnar, A membrane micropump electrostatically actuated across the working fluid, J. Micromechanics Microengineering. 15 (2005) 2309-2316. doi:10.1088/0960-1317/15/12/013.

[13] K.S. Lee, B. Kim, M.A. Shannon, An electrostatically driven valve-less peristaltic micropump with a stepwise chamber, Sensors Actuators A Phys. 187 (2012) 183-189. doi:10.1016/J.SNA.2012.08.040.

[14] B. Kim, Electrostatically driven micropump with peristaltically moving membrane, Micro \&amp; Nano Lett. 8 (2013) 654-658. doi:10.1049/mnl.2013.0328.

[15] H. Kim, A.A. Astle, K. Najafi, L.P. Bernal, P.D. Washabaugh, An Integrated Electrostatic Peristaltic 18-Stage Gas Micropump With Active Microvalves, J. Microelectromechanical Syst. 24 (2015) 192-206. doi:10.1109/JMEMS.2014.2327096.

[16] I. Lee, P. Hong, C. Cho, B. Lee, K. Chun, B. Kim, Four-electrode micropump with peristaltic motion, Sensors Actuators A Phys. 245 (2016) 19-25. doi:10.1016/J.SNA.2016.04.010.

[17] J. Santos, B. Lopes, P.J.C. Branco, Ionic polymer-metal composite material as a diaphragm for micropump devices, Sensors Actuators A Phys. 161 (2010) 225-233. doi:10.1016/J.SNA.2010.04.032.

[18] D.N.C. Nam, K.K. Ahn, Design of an IPMC diaphragm for micropump application, Sensors Actuators A Phys. 187 (2012) 174-182. doi:10.1016/J.SNA.2012.08.027.

[19] F.A. Mohd Ghazali, C.K. Mah, A. AbuZaiter, P.S. Chee, M.S. Mohamed Ali, Soft dielectric elastomer actuator micropump, Sensors Actuators, A Phys. 263 (2017) 276- 
284. doi:10.1016/j.sna.2017.06.018.

[20] M. Shikida, K. Sato, Micromachined S-shaped Actuator, in: Sixth Int. Symp. Micro Mach. Hum. Sci., 1995: pp. 167-172.

[21] J. Oberhammer, G. Stemme, Design and fabrication aspects of an S-shaped film actuator based DC to RF MEMS switch, J. Microelectromechanical Syst. 13 (2004) 421428. doi:10.1109/JMEMS.2004.828723.

[22] J.R. Frutos, D. Vernier, F. Bastien, M. De Labachelerie, Y. Bailly, An electrostatically actuated valve for turbulent boundary layer control, Proc. IEEE Sensors. 2005 (2005) 82-88. doi:10.1109/ICSENS.2005.1597642.

[23] K. SATO, Driving Voltage Reduction of a Micromechanical Optical Switch Driven by Electrostatic Force Using an S-Shaped Deformable Thin-Film Mirror, J. Adv. Mech. Des. Syst. Manuf. 7 (2013) 410-421. doi:10.1299/jamdsm.7.410.

[24] B. BERGE, Electrostatically Actuated Device, WO 2018/041866, 2018. https://patentscope.wipo.int/search/en/detail.jsf?docld=WO2018041866\&recNum=\& maxRec $=1000$ \&office $=$ \&prevFilter $=\&$ sortOption=\&queryString $=\&$ tab $=$ PCTBiblio.

[25] J. Jarosz, Q. Lavigne, N. Molliex, G. Chenon, G. Noetinger, D.-D. Tran, B. Berge, Experimental optical analysis of an original presbyopia-correcting variable focus lens, 2018. doi:10.13140/RG.2.2.30649.88161.

[26] M.W. Ashraf, S. Tayyaba, N. Afzulpurkar, Micro Electromechanical Systems (MEMS) based microfluidic devices for biomedical applications, Int. J. Mol. Sci. 12 (2011) 36483704. doi:10.3390/ijms12063648.

[27] B.J. Kim, E. Meng, Micromachining of Parylene C for bioMEMS, Polym. Adv. Technol. 27 (2016) 564-576. doi:10.1002/pat.3729.

[28] A. Kahouli, A. Sylvestre, L. Ortega, F. Jomni, B. Yangui, M. Maillard, B. Berge, J.C. Robert, J. Legrand, Structural and dielectric study of parylene $C$ thin films, Appl. Phys. Lett. 94 (2009) 152901. doi:10.1063/1.3114404. 


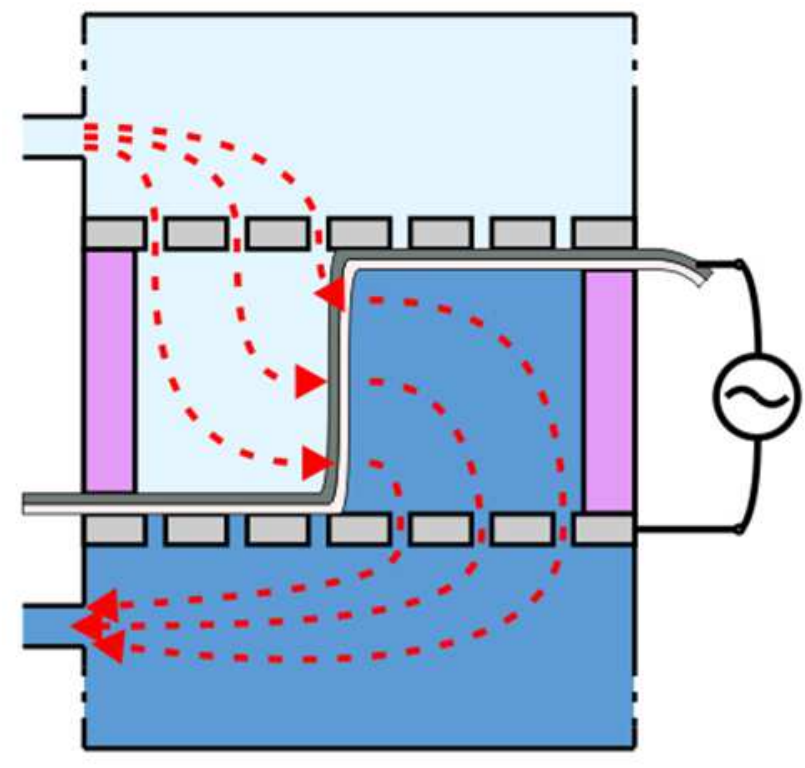

A novel concept of microfluidic actuator based on a slack metallized film confined between two parallel grid-electrodes separated by a gap is presented. The film is loosely attached such that it can move freely between the two grid-electrodes. Upon voltage application between the metallized film and one gridelectrode, electrostatic attraction tends to press the metallized film on the grid, thus pushing the fluid through the grid. This evolution happens through a film fold travelling across the cell width. Samples using a replication of grid-electrodes manufactured by silicon anisotropic etching have been assembled. An equilibrium theory of operation and an experimental characterization with measurements of exchanged liquid volumes, differential pressures, capacitance and applied voltage are presented, both agreeing within $+/-15 \%$. It will be shown that actuators with an active zone of $8 \mathrm{~mm} \times 45 \mathrm{~mm}$ and a gap of $0.25 \mathrm{~mm}$ can move $100 \mu$ lagainst differential pressures of about $200 \mathrm{~Pa}$ with a $100 \mathrm{~V}$ power supply at $100 \mathrm{~Hz}$ consuming few tens of $\mathrm{mW}$. The only moving part is the deformable film and the actuator is silent when filled with liquids. The new concept also supports an accurate capacitance monitoring of the exchanged volumes. The actuation can be reversible by connecting one or the other grid electrode. This new pumping mechanism has been primarily designed for exchanging a given volume of two insulating fluids of different refractive indices, in order to activate ophthalmic variable eyeglasses for presbyopia correction. Nevertheless, the same concept can be extended to continuous flow pumping. 